\title{
Performance of OptiMAL ${ }^{\circledR}$ in the Diagnosis of Plasmodium vivax and Plasmodium falciparum Infections in a Malaria Referral Center in Colombia
}

\author{
Beatriz E Ferro/+ , Iveth J González, Fanny de Carvajal*, Gloria I Palma*, Nancy G Saravia
}

Centro Internacional de Entrenamiento e Investigaciones Médicas, Avenida 1 Norte No. 3-03, Cali, Colombia *Laboratorio de

Parasitología y Servicio de Diagnóstico de Malaria, Departamento de Microbiología, Universidad del Valle, Cali, Colombia

Alternative, non-microscopic methods for the diagnosis of malaria have recently become available. Among these, rapid dipstick methods stand out. One such test, OptiMAL ${ }^{\circledR}$, is based on the immunochromatographic detection of Plasmodium lactate dehydrogenase $(p L D H)$ and has the capacity to detect and distinguish infections caused by P. falciparum and Plasmodium sp. This capacity is particularly important in countries where different species of Plasmodium co-exist. In this study we evaluated the performance of OptiMAL ${ }^{\circledR}$ in an urban referral center for malaria diagnosis. Two sets of patients were included: one $(n=112)$ having predetermined infections with $\mathrm{P}$. falciparum or P. vivax and individuals with negative blood smears; and another consisting of all eligible consecutive patients $(n=80)$ consulting for diagnosis at the referral center during one month. The overall diagnostic efficiency of OptiMAL ${ }^{\circledR}$ for both sets of patients was $96.9 \%$. Efficiency was higher for $\mathrm{P}$. vivax (98.1\%) than for $\mathrm{P}$. falciparum $(94.9 \%)$. These results corroborate the diagnostic utility of OptiMAL ${ }^{\circledR}$ in settings where $\mathrm{P}$. vivax and $\mathrm{P}$. falciparum coexist and support its implementation where microscopic diagnosis is unavailable and in circumstances that exceed the capacity of the local microscopic diagnosis facility.

Key words: rapid diagnosis - malaria - OptiMAL ${ }^{\circledR}$ - dipstick - Colombia

Rapid and accurate diagnosis is critical to the effective management of malaria and is one of the principal interventions in the control of this disease (WHO 1993). Better utilization of microscopy, the standard method of diagnosis, and the development of alternative diagnostic methods, could substantially improve the control of malaria (WHO 2000).

Microscopic diagnosis is highly sensitive and specific. This test allows discrimination of asexual and sexual parasite stages, differentiation of the four species that cause human malaria, and quantification of parasitemia. However, requirements such as electricity, a functioning microscope, highly trained technicians, and strict quality control, result in this method being unavailable in remote endemic regions.

In places where microscopic diagnosis is not available, the diagnosis of malaria is based on the clinical presentation of illness. This practice is unreliable because of the non-specific nature of malaria symptoms, which can readily be confused with those of other tropical febrile illnesses (WHO 2000). Reliance on clinical diagnosis results in inappropriate treatment of patients not having

This investigation was supported by the UNDP/World Bank/ WHO Special Programme for Research and Training in Tropical Diseases, Project ID 981015, and the Laboratorio de Parasitología, Departamento de Microbiología, Universidad del Valle and Corporación CIDEIM.

${ }^{+}$Corresponding author. Fax: +57-2-6672989. E-mail: cideim@cideim.org.co

Received 25 September 2001

Accepted 24 April 2002 malaria with antimalarial drugs, self-medication and incomplete treatment upon improvement of symptoms. All of these practices could favor the development of drug resistance.

Notwithstanding the obvious virtues of microscopy, alternative methods of malaria diagnosis that overcome its limitations are needed. In response to this need, several new diagnostic methods have been developed including serological tests, PCR (polymerase chain reaction), and fluorescent microscopy like $\mathrm{QBC}^{\circledR}$ (quantitative buffy coat) (Makler et al. 1998). Although these technologies have provided alternatives for some diagnostic circumstances, the requirement of sophisticated equipment and training has prevented the displacement of microscopy as the routine diagnostic method.

More recently, immunochromatographic tests, also known as rapid diagnostic tests of malaria, have been developed. The histidine rich protein II (HRPII) (Shiff et al. 1993) and Plasmodium lactate dehydrogenase (pLDH) (Makler \& Hinrichs 1993), are the most common antigens used. OptiMAL ${ }^{\circledR}$ dipstick test (Flow Inc., Portland, OR) is able to differentiate between $P$. falciparum and non $P$. falciparum malaria based on the ability of monoclonal antibodies to react with Plasmodium sp. and P. falciparum specific epitopes of pLDH. In countries where different species of Plasmodium co-exist, the ability to detect Plasmodium sp. infections is an advantage of OptiMAL ${ }^{\circledR}$ over other currently available rapid diagnostic tests that only detect P. falciparum.

In Colombia during the past five years, approximately $55 \%$ of malaria cases were caused by $P$. vivax, $45 \%$ by $P$. falciparum and a low proportion (less than $0.1 \%$ overall), by $P$. malariae, (Ministerio de Salud 1999, Vergara et al. 2001). Treatment regimens for $P$. falciparum differ from 
those for other species of Plasmodium because of the high frequency of resistance to chloroquine (Osorio et al. 1999) the absence of hipnozoite liver stages and the risk of lethal complications of infection. Therefore, diagnostic tests that both detect and distinguish P. falciparum and Plasmodium sp. infection are essential to the appropriate treatment and management of malaria in Colombia.

In this study we have evaluated the rapid diagnostic test OptiMAL ${ }^{\circledR}$ in a referral center under the conditions of a public malaria diagnostic service. Sensitivity, specificity, predictive values and efficiency in comparison with conventional microscopy as "gold standard" were determined. Additionally, PCR was used as a second gold standard because of its greater sensitivity in cases of low parasitemia (Snounou et al. 1993).

\section{MATERIALS AND METHODS}

Study site - This study was conducted in Cali, the second largest city in Colombia, within the Malaria Diagnostic Service of the Universidad del Valle and the Secretaria de Salud Pública Municipal. Although malaria is not transmitted in the city of Cali, this urban center is located near endemic areas and serves as referral center for malaria diagnosis in South Western Colombia.

Patients and samples - Patients consulting the Malaria Diagnostic Service were invited to participate in the study if they had fever and a history of traveling to an endemic area. Informed written consent was obtained from all patients included. A first study group, designated Group I, was composed to conduct an exploration of the test performance in malaria patients with $P$. falciparum and $P$. vivax, and in controls having negative thick smears. The second group, Group II, consisted of consecutive patients consulting the Malaria Diagnostic Service during a period of one month. The latter group was enrolled in order to evaluate the performance of the diagnostic for the case mix (including a high proportion of non-malarial febrile illness) typically consulting this service. Thick smear and OptiMAL ${ }^{\circledR}$ were performed for both groups. In addition, PCR was performed for Group II. A fingerprick blood sample was obtained from each patient and was used to prepare thick and thin smears and to fill a heparinized capillary tube used for the OptiMAL ${ }^{\circledR}$ test. Additionally, for Group II blood samples were collected on Whatman filter paper \#3 for DNA extraction.

Diagnosis by thick/thin smear - Thick smears were treated with methylene blue to remove hemoglobin and thereby facilitate reading of the slides, then stained with $1 \%$ Giemsa in phosphate buffer ( $\mathrm{pH}$ 7.2). Thin smears were fixed in methanol and stained with $2 \%$ Giemsa and were evaluated only if parasite species confirmation was needed. All the smears were evaluated using light microscopy at 1000X magnification by two trained medical technologists with more than a year of experience in reading malaria slides. A thick smear was considered negative if no parasites were seen in at least 200 fields. In positive smears parasitemia was calculated as follows:

$\frac{\text { Number of parasites }}{100 \text { leukocytes }} \times 8000$ leukocytes $/ \mu \mathrm{l}=$ parasites $/ \mu \mathrm{l}$
Diagnosis using OptiMAL ${ }^{\circledR}$ - The OptiMAL ${ }^{\circledR}$ test (Flow Inc. Portland, OR) employs immunochromatography in a dipstick that contains monoclonal antibodies (Mabs) directed against the enzyme pLDH. There are two Mabs: one is specific for P. falciparum and is located at the bottom of the dipstick and the other is specific for Plasmodium sp. and is located in the middle of the dipstick. A polyclonal goat anti-mouse antibody located at the top of the dipstick functions as a reaction control by capturing the mouse anti-pLDH monoclonal colloidal gold conjugate. The whole process was conducted following the manufacturer's instructions. In accordance with the species prevalent in Colombia, positive results to Plasmodium sp. were considered to be $P$. vivax in our analysis.

\section{PCR}

DNA extraction - DNA was extracted from the blood spots impregnated on filter paper using a modified Saponin-Chelex protocol (Giraldo et al. 1998). The blood spots were cut up into small pieces, suspended in $1 \%$ Saponin in distilled water (Sigma, St. Louis, MO) and incubated on ice to produce lysis of the cells, followed by a second incubation at $56^{\circ} \mathrm{C}$ and $100^{\circ} \mathrm{C}$ with $5 \%$ Chelex-100 resin (BioRad, Hercules, CA) that captures and releases the DNA in response to the change of temperature.

Nested diagnostic PCR - A nested diagnostic PCR was used as described previously (Snounou et al. 1993). This PCR is based on the amplification of the sequences of the gene that code for the $18 \mathrm{~S}$ subunit of the four malaria parasites. Ten microliters of the extracted DNA were used as template for the first amplification reaction. Five microliters of the amplification product of the first reaction were used for the second amplification. All the amplifications were performed in a PTC-100 thermocycler (MJ Research Inc.) and the DNA amplified by PCR was electrophoresed in $2 \%$ agarose gels and visualized on a UV transiluminator after staining with ethidium bromide.

Data analysis - The sensitivity, specificity and predictive values of the OptiMAL ${ }^{\circledR}$ test were calculated using the thick smear as the gold standard. For Group II both the thick smear and PCR were independently used as gold standards. Overall, diagnostic efficiency of OptiMAL ${ }^{\circledR}$ for malaria and for each species of Plasmodium was determined based on the combined results of Group I and II, as described by Weigle et al. (1993) using the formula:

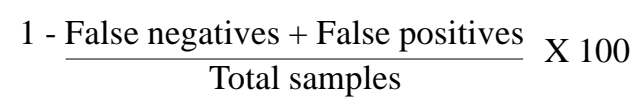

The statistical analyses were conducted with SPSS 7.5 for Windows ${ }^{\circledR}$ and Epi-Info 6.04 .

\section{RESULTS}

Diagnosis with thick smear and OptiMAL ${ }^{\circledR}$ in Group $I$ - A total of 112 patients were included in Group I. Eighty-seven patients presented a positive thick smear: 55 with $P$. vivax, 28 with $P$. falciparum, 3 with mixed malaria (P. vivax and P. falciparum), 1 with P. malariae; while 25 patients were negative for malaria. Sensitivity was higher for $P$. vivax than $P$. falciparum, whereas specificity was comparable for both species (Table I). 
TABLE I

Sensitivity, specificity and positive (PPV) and negative (NPV) predictive values of OptiMAL ${ }^{\circledR}$ for the diagnosis of Plasmodium falciparum or $P$. vivax infections in Group $\mathrm{I}^{a}$

\begin{tabular}{lcc}
\hline & \multicolumn{2}{c}{ Group I } \\
\cline { 2 - 3 } & P.falciparum & P. vivax \\
\hline \% sensitivity (95\%IC) & $89.3(70.6-97.2)$ & $98.2(89.0-99.9)$ \\
\% specificity (95\%IC) & $100(84.3-100)$ & $96.0(77.7-99.8)$ \\
\% PPV (95\%IC) & $100(83.4-100)$ & $98.2(89.0-99.9)$ \\
\% NPV (95\%IC) & $89.3(70.6-97.2)$ & $96.0(77.7-99.8)$ \\
\hline
\end{tabular}

$a$ : composed to conduct an exploration, a total of 112 patients were included.

Among cases of $P$. falciparum infection, there were three false negative tests in which OptiMAL ${ }^{\circledR}$ was positive but failed to correctly diagnose the species. In two of these cases the level of parasitemia was low (80 and 480 parasites $/ \mu \mathrm{l}$ ) and in one the parasitemia consisted only of sexual forms. Likewise, for $P$. vivax cases the only false negative case presented scarce parasitemia ( 80 parasites/ $\mu \mathrm{l})$. A false positive result for $P$. vivax occurred in a patient with a history of travel to an endemic area but negative thick smear on two occasions.

In the three cases of mixed P. falciparum/P.vivax malaria, OptiMAL ${ }^{\circledR}$ diagnosed only P. falciparum in two and $P$. vivax in one. In the single case of $P$. malariae, reactivity occurred with the Mab for Plasmodium sp., and therefore it was considered a $P$. vivax infection in our analysis.

Diagnosis using thick smear, OptiMAL ${ }^{\circledR}$ and PCR in Group II - During the sampling period (one month), 80 patients were included in this group. Fifteen samples were positive for $P$. vivax, four for P. falciparum, one had mixed infection and the remaining 60 samples were negative for malaria by thick smear. All the samples that were positive by thick smear in this group were also positive by OptiMAL ${ }^{\circledR}$ for the corresponding species. Sensitivity and specificity was high for both species but the low prevalence of malaria infection resulted in wide confidence intervals and false positive results for $P$. falciparum affected the corresponding positive predictive value (Table II).

In this group the performance of OptiMAL ${ }^{\circledR}$ was also analyzed using PCR as the reference standard. In this analysis the sensitivity of OptiMAL ${ }^{\circledR}$ for both species was lower; $83.3 \%$ for P. falciparum and $84.2 \%$ for $P$. vivax versus $100 \%$ using the conventional gold standard. The specificity was unaffected (data not shown). For $P$. falciparum the single false negative sample came from a patient in post-treatment control. For $P$. vivax there were three false negative cases and two were from patients with history of previous episodes of malaria.

The predetermined diagnosis of patients included in Group I and the small number of patients with malaria in Group II $(n=20)$ limited the generalizability of the results that were obtained. Therefore, we conducted an analysis of Group I and Group II combined to obtain a more representative assessment of the diagnostic performance of OptiMAL ${ }^{\circledR}$ compared with thick smear (Table III). The results were similar to those obtained for Group I, but confidence intervals were narrower. Overall, diagnostic efficiency for malaria was $96.9 \%$. The diagnostic efficiency for $P$. vivax was higher $(98.1 \%)$ than for P. falciparum (94.9\%).

Parasitemia - Parasitemia levels for P. falciparum in Group I varied from 80 to 64,880 parasites/ $\mu$ land for $P$. vivax from 80 to 28,800 parasites/ $\mu$ l. The three false negative cases of $P$. falciparum using OptiMAL ${ }^{\circledR}$ had parasitemia values ranging from 80 to 800 parasites/ $\mu 1$ and the false negative of $P$. vivax had a parasitemia of 80 parasites/ $\mu$ l. In Group II parasitemia varied from 80 to 20,720 parasites/ $\mu$ l for $P$. falciparum and from 160 to 20,320 parasites $/ \mu$ for $P$. vivax.

TABLE II

Sensitivity, specificity and positive (PPV) and negative (NPV) predictive values of OptiMAL ${ }^{\circledR}$ for the diagnosis of Plasmodium falciparum or P. vivax infections in Group II ${ }^{a}$

\begin{tabular}{lcr}
\hline & \multicolumn{2}{c}{ Group II } \\
\cline { 2 - 3 } & P.falciparum & P. vivax \\
\hline \% sensitivity (95\%IC) & $100(39.6-100)$ & $100(75.9-100)$ \\
\% specificity (95\%IC) & $95.0(85.2-98.7)$ & $98.0(89.9-99.9)$ \\
\% PPV (95\%IC) & $57.1(20.0-88.2)$ & $94.1(69.2-99.7)$ \\
\% NPV (95\%IC) & $100(92.1-100)$ & $100(92.4-100)$
\end{tabular}

$a$ : consecutive patients consulting the Malaria Diagnostic Service during a period of one month, a total of 80 patients were included.

TABLE III

Sensitivity, specificity and positive (PPV) and negative (NPV) predictive values and efficiency of OptiMAL ${ }^{\circledR}$ for the diagnosis of Plasmodium falciparum or $P$. vivax infections in Groups I and II combined

\begin{tabular}{lccr}
\hline & \multicolumn{3}{c}{ Groups I + II } \\
\cline { 2 - 4 } & P.falciparum & P. vivax & Malaria overall \\
\hline \% sensitivity (95\%IC) & $90.6(73.8-97.5)$ & $98.6(91.3-99.9)$ & $99.1(94.2-100)$ \\
$\%$ specificity (95\%IC) & $96.5(89.3-99.1)$ & $97.6(91.0-99.6)$ & $94.1(86.2-97.8)$ \\
$\%$ PPV (95\%IC) & $90.6(73.8-97.5)$ & $97.2(89.4-99.5)$ & $95.5(89.5-98.3)$ \\
\% NPV (95\%IC) & $96.5(89.3-99.1)$ & $98.8(92.6-99.9)$ & $98.8(92.4-99.9)$ \\
\hline
\end{tabular}




\section{DISCUSSION}

In this study the OptiMAL ${ }^{\circledR}$ test efficiently diagnosed malaria caused by either P. vivax or P. falciparum. However, the sensitivity and efficiency of the test was lower for $P$. falciparum infections. This lower efficiency of OptiMAL ${ }^{\circledR}$ for $P$. falciparum detection was due to false positive tests in a small number of cases. Studies conducted in settings having a high prevalence of $P$. falciparum have shown OptiMAL ${ }^{\circledR}$ to be highly sensitive and specific for infections having parasitemias $>500$ parasites/ $\mu \mathrm{l}$ (Hunt-Cooke at el. 1999). On the other hand, disparity in the sensitivity for $P$. vivax $(94 \%)$ and $P$. falciparum (88\%) infections has also been reported by Palmer et al. (1998) in a study conducted in Honduras. Characteristics of transmission such as intensity, endemicity of multiple species of Plasmodium, acquired resistance in the exposed population and treatment, can individually and collectively influence the results of this and other diagnostic tests for malaria. Hence, these variables need to be considered in the interpretation of diagnostic tests and management of the corresponding patients.

PCR was also used as reference standard to evaluate the OptiMAL ${ }^{\circledR}$ yielding a lower sensitivity than that obtained with thick smear. PCR detected more positive cases than thick smear or OptiMAL ${ }^{\circledR}$ because it detects the parasite DNA, which could be present in either dead or live parasites, whereas OptiMAL ${ }^{\circledR}$ detects an enzyme produced by live parasites. The fact that $\mathrm{pLDH}$ is present only when there are living parasites in the sample is especially relevant for post-treatment evaluations (Makler et al. 1998).

Although there were only four false negative cases in this study, these presented some characteristics that could influence OptiMAL ${ }^{\circledR}$ sensitivity including low parasitemia, gametocytemia without asexual forms and the apparent effects of treatment on parasite metabolism including $\mathrm{pLDH}$. The presence of low parasitemias, especially below the detection level that has been established by the manufacturer as $100-200$ parasites/ $\mu 1$, was associated with false negative results in this study. Gametocytes produce $\mathrm{pLDH}$, but since gametocyte parasitemias are usually low, they are not detected efficiently by OptiMAL ${ }^{\circledR}$ (Moody et al. 2000). Two of the false negative cases in this study were from patients that had received previous treatment. OptiMAL ${ }^{\circledR}$ sensitivity has been shown to be influenced by the decrease of parasitemia and pLDH levels after treatment (Moody et al. 2000).

In all false negative cases of P. falciparum, OptiMAL ${ }^{\circledR}$ yielded a positive result with the monoclonal antibody for Plasmodium sp., which was interpreted as $P$. vivax infection. In cases of low or gametocyte parasitemias, $P$. falciparum $\mathrm{pLDH}$ may react only with the genus-specific antibody as has been described (Hunt-Cooke et al. 1998). This fact should be taken into consideration in regions where $P$. falciparum and $P$. vivax coexist since treatment regimens are different for these species.

During this study only one patient presented $P$. malariae infection, which by OptiMAL ${ }^{\circledR}$ was positive with the Mab for Plasmodium sp. In previous studies it has been observed that OptiMAL ${ }^{\circledR}$ is less efficient in de- tecting P. malariae and P. ovale (Srinivasan et al. 2000). In Colombia $P$. malariae is becoming an increasingly important species, especially in some regions of the Pacific Coast where up to $10 \%$ of infections may be caused by this species (Vergara et al. 2001). Overall $0.1 \%$ malaria infections in Colombia are due to P. malariae (Ministerio de Salud 1999).

Mixed infections account for $1 \%$ of malaria cases in Colombia (Ministerio de Salud 1999). In cases of mixed P. falciparum/P. vivax malaria, OptiMAL ${ }^{\circledR}$ presents reactions with both the P. falciparum and Plasmodium sp. Mabs, a pattern which corresponds to diagnosis of $P$. falciparum infection. Therefore, the actual format of OptiMAL ${ }^{\circledR}$ favors the diagnosis of P. falciparum but masks co-infection by $P$. vivax or other species of Plasmodium and hence constitutes a limitation of the current configuration of the test in settings of co-endemicity (Srinivasan et al. 2000).

Our results, which are similar to those obtained in previous studies in other endemic and non-endemic regions, indicate that OptiMAL ${ }^{\circledR}$ would be useful and should be implemented in areas of transmission and circumstances not covered by the traditional microscopic diagnosis of malaria. These situations include epidemic outbreaks, occupationally exposed groups, and displaced populations moving from or toward endemic areas and therefore represent a risk of spreading malaria. All of these circumstances are common in Colombia and in other developing countries. However, some issues of rapid diagnosis of malaria by OptiMAL ${ }^{\circledR}$ and other tests are still unresolved. The applicability of such tests in assessing treatment efficacy, and the cost-effectiveness of the implementation of these alternative methods have neither been extensively nor definitively investigated. Furthermore, efforts to develop a more sensitive test that can detect all the four species of Plasmodium and quantify the parasite burden should continue.

\section{ACKNOWLEDGMENTS}

To Rafael Tovar for assistance with the statistical analysis, to Meleny Ramírez and Jorge Iván Zapata for their assistance in sample and data collection and to the Servicio de Diagnóstico de Malaria de la Secretaria de Salud de Cali for facilitating the conduction of this study.

This article is dedicated to the memory of Luis Ernesto Giraldo, MD PhD, who was the mentor of this research.

\section{REFERENCES}

Giraldo LE, Acosta MC, Labrada LA, Praba A, MontenegroJames S, Saravia N, Krogstad D 1998. Frequency of the ASN-108 and THR-108 point mutations in the dihydrofolate reductase gene in Plasmodium falciparum from southwest Colombia. Am J Trop Med Hyg 59: 124-128.

Hunt-Cooke A, Chiodini P, Doherty T, Moody A, Ries J, Pinder M 1999. Comparison of parasite lactate dehydrogenasebased immunochromatographic antigen detection assay (OptiMAL ${ }^{\circledR}$ ) with microscopy for the detection of malaria parasites in human blood samples. Am J Trop Med Hyg 60: 173-176.

Makler MT, Hinrichs DJ 1993. Measurement of the lactate dehydrogenase activity of Plasmodium falciparum as an assessment of parasitemia. Am J Trop Med Hyg 48: 205210. 
Makler MT, Palmer CJ, Ager AL 1998. A review of practical techniques for the diagnosis of malaria. Ann Trop Med Parasitol 92: 419-433.

Ministerio de Salud 1999. Departamento de Epidemiología, Campañas Directas, Registro de casos de malaria a nivel nacional y por departamento 1990-1999.

Moody A, Hunt-cooke A, Gabbett E, Chiodini P 2000. Performance of the OptiMAL ${ }^{\circledR}$ malaria antigen capture dipstick for malaria diagnosis and treatment monitoring at the hospital for tropical diseases, London. British J Haematol 109: $1-5$.

Osorio LE, Giraldo LE, Grajales LF, Arriaga AL, Andrade AL, Barat L, Trenton R 1999. Assessment of therapeutic response of Plasmodium falciparum to chloroquine and sulfadoxine-pyrimethamine in an area of low malaria transmission in Colombia. Am J Trop Med Hyg 61: 968-972.

Palmer, CJ, Lindo JF, Klaskala W, Quesada J, Kaminsky R, Ager AL 1998. Evaluation of the OptiMAL test for rapid diagnosis of Plasmodium vivax and Plasmodium falciparum. J Clin Microbiol 36: 203-206.

Shiff CJ, Premji Z, Minjas ZN 1993. The rapid manual ParasightF test. A new diagnostic tool for Plasmodium falciparum infection. Trans R Soc Trop Med Hyg 87: 646-648.
Snounou G, Viriyakosol S, Ping X, Jarra W, Pinheiro L, do Rosario VE, Thaithong S, Brown KN 1993. High sensitivity detection of human malaria parasites by the use of nested polymerase chain reaction. Mol Biochem Parasitol 61: 315320.

Srinivasan S, Moody AH, Chiodini PL 2000. Comparison of blood-film microscopy, the OptiMAL ${ }^{\circledR}$ dipstick, Rhodamine-123 fluorescence staining and PCR, for monitoring antimalarial treatment. Ann Trop Med Parasitol 94: 227-232.

Vergara J, Hurtado S, Alvarez VH, Arévalo M, Herrera S 2001. Caracterización de la transmisión de Plasmodium malariae en cuatro regiones colombianas endémicas de malaria. Biomédica 21: 53-61.

Weigle KA, Escobar M, Arias AL, Martinez F, Rojas C 1993. A clinical prediction rule for american cutaneous leishmaniasis in Colombia. Int J Epidemiol 21: 548-558.

WHO-World Health Organization 1993. A Global Strategy for Malaria Control, WHO, Geneva.

WHO-World Health Organization 2000. New perspectives: malaria diagnosis. Report of a joint WHO/USAID informal consultation, 25-27 October 1999, WHO/CDS/RBM/ 2000.14 and WHO/MAL/2000.1091, Geneva. 
